\title{
STURMIAN THEOREMS AND POSITIVE RESOLVENTS
}

\author{
BY \\ KURT KREITH
}

1. Introduction. The classical Sturmian theorem of ordinary differential equations deals with functions $u(x)$ and $v(x)$ which are, respectively, solutions of differential equations

$$
\begin{aligned}
L u & \equiv-\frac{d}{d x}\left(a \frac{d u}{d x}\right)+c u=0, \\
M v & \equiv-\frac{d}{d x}\left(\alpha \frac{d v}{d x}\right)+\gamma v=0 .
\end{aligned}
$$

Under the assumption that " $L$ is larger than $M$ " (in the sense that $a(x) \geqq \alpha(x)>0$ and $c(x) \geqq \gamma(x)$ ) one can infer information about all solutions of (2) from knowledge about a particular nontrivial solution of (1)-i.e. if $u\left(x_{1}\right)=u\left(x_{2}\right)=0$ then every solution of (2) has a zero in $\left[x_{1}, x_{2}\right]$.

These ideas have been generalized to second order elliptic equations by several authors ([1]-[4]) considering elliptic operators

$$
L u \equiv-\sum \frac{\partial}{\partial x_{j}}\left(a_{i j} \frac{\partial u}{\partial x_{i}}\right)+c u, \quad M v=-\sum \frac{\partial}{\partial x_{j}}\left(\alpha_{i j} \frac{\partial v}{\partial x_{i}}\right)+\gamma v,
$$

and also by Protter [5] and Swanson [6] considering the nonselfadjoint case. Given a proper relation among the coefficients of $L$ and $M$ and that $L u=0$ has a nontrivial solution with nodal domain $\Omega$, then it can be shown that every solution of $M v=0$ has a zero in $\bar{\Omega}$. While all the above proofs of this fact make essential use of some sort of ordering among elliptic operators, the nature of this ordering is never defined in operator-theoretic terms.

The results of $\S 2$ below suggest that it is an order relationship between certain resolvents of the differential operators $L$ and $M$ which underlies the separation properties characteristic of Sturmian theorems. It will be shown that quite general operator equations in a Banach space $\mathscr{B}$ satisfy a type of Sturmian theorem if the operators' resolvents satisfy prescribed positivity requirements with respect to a cone $\mathscr{P}$.

In order to apply this theory to differential operators, one must first establish the corresponding positivity properties for their resolvents. This is done in $\$ 3$ for sufficiently regular nonselfadjoint second order elliptic operators, and the general theory of $\$ 2$ is then applied in the proof of two Sturmian theorems and the establishment of criteria for certain Green's functions to be positive.

Received by the editors January 2, 1968 and, in revised form, June 21, 1968. 
2. An abstract Sturmian theorem. Our abstract formulation of a Sturmian theorem will be valid in a real Banach space $\mathscr{B}$ equipped with a reproducing cone $\mathscr{P}$ of "nonnegative" elements.

2.1. Definition. A closed subset $\mathscr{P}$ of $\mathscr{B}$ is called a reproducing cone if it satisfies

(i) $u \in \mathscr{P}$ and $v \in \mathscr{P} \Rightarrow u+v \in \mathscr{P}$;

(ii) $u \in \mathscr{P}$ and $\alpha \geqq 0 \Rightarrow \alpha u \in \mathscr{P}$;

(iii) $u \in \mathscr{P}$ and $-u \in \mathscr{P} \Rightarrow \boldsymbol{u}=\theta$;

(iv) $w \in \mathscr{B} \Rightarrow \exists u, v \in \mathscr{P} \ni w=u-v$.

If $u-v \in \mathscr{P}$, we write $u \geqq v$. A linear operator $B$ mapping $\mathscr{B}$ into $\mathscr{B}$ is called positive if it maps $\mathscr{P}$ into $\mathscr{P}$. Under additional hypotheses, certain positive operators have a remarkable property which will be referred to as property $(*)$ : the operator $B$ has exactly one (normalized) eigenvector in $\mathscr{P}$, and the corresponding eigenvalue is simple, positive, and larger than the absolute value of any other eigenvalue. The criterion for property $\left({ }^{*}\right)$ which will be most useful here is based on the concept of $u_{0}$-positivity.

2.2. Definition. We say that a bounded linear operator $B$ is $u_{0}$-positive if there exists a nonzero $u_{0} \in \mathscr{P}$ with the following property: for every nonzero $u \in \mathscr{P}$ there exists a positive number $\delta$ such that $\delta^{-1} u_{0} \leqq B u \leqq \delta u_{0}$.

The following two theorems are found in [8], which also has an extensive discussion of positive operators.

2.3. THEOREM. If $B$ is compact and $u_{0}$-positive, then $B$ has property $\left({ }^{*}\right)$.

Proof. See [8, pp. 76 and 81$]$.

2.4. THEOREM. If $B$ is compact and $u_{0}$-positive and $v_{0}$ is the normalized positive eigenvector of $B$ prescribed by $(*)$, then $B$ is $v_{0}$-positive.

Proof. See [8, p. 76].

Our generalized comparison theorem depends on the following result.

2.5. LemMA. Let $B$ be a compact $u_{0}$-positive operator and $\beta$ and $v_{0}$ be the maximal eigenvalue and corresponding normalized positive eigenfunction prescribed by $\left(^{*}\right)$. If there exists $a u \in \mathscr{P}(u \neq \theta)$ such that $B u-\lambda u \in \mathscr{P}$ then $\lambda \leqq \beta$. If $\lambda=\beta$, then $u$ is a scalar multiple of $v_{0}$.

Proof. Since $\beta>0$, it is sufficient to deal with the case $\lambda>0$. Recalling that $\mathscr{P}$ is closed by definition, we define

$$
\varepsilon_{0}=\sup \left\{\varepsilon \mid B\left(v_{0}-\varepsilon u\right) \in \mathscr{P}\right\} .
$$

From $u \in \mathscr{P}(u \neq \theta)$ and the $v_{0}$-positivity of $B$ it follows that $0<\varepsilon_{0}<\infty$. Writing

$$
B\left(v_{0}-\varepsilon_{0} u\right)=\beta v_{0}-\varepsilon_{0} B u=\beta v_{0}-\varepsilon_{0}[\lambda u+(B u-\lambda u)]
$$

we get

$$
B\left[B\left(v_{0}-\varepsilon_{0} u\right)+\varepsilon_{0}(B u-\lambda u)\right]=\beta B\left(v_{0}-\varepsilon_{0} \frac{\lambda}{\beta} u\right)
$$


By hypothesis the left side of (3) belongs to $\mathscr{P}$, implying that $B\left(v_{0}-\varepsilon_{0}(\lambda / \beta) u\right) \in \mathscr{P}$. From the definition of $\varepsilon_{0}$ it follows that $\lambda \leqq \beta$.

In case $\lambda=\beta$, (3) yields

$$
\beta^{-1} B^{2}\left(v_{0}-\varepsilon_{0} u\right) \leqq B\left(v_{0}-\varepsilon_{0} u\right) .
$$

If $v_{0}-\varepsilon_{0} u \neq \theta$, the $v_{0}$-positivity of $B$ implies the existence of a $\delta>0$ such that

$$
\beta^{-1} \delta^{2} v_{0} \leqq \beta^{-1} B^{2}\left(v_{0}-\varepsilon_{0} u\right) \leqq B\left(v_{0}-\varepsilon_{0} u\right)=\beta v_{0}-\varepsilon_{0} B u .
$$

But by the $v_{0}$-positivity of $B$, this contradicts the maximal property of $\varepsilon_{0}$, and therefore $v_{0}=\varepsilon_{0} u$ whenever $\lambda=\beta$.

2.6. COMPARISON THEOREM. Let $L$ and $M$ be (not necessarily bounded) linear operators in $\mathscr{B}$ and suppose there exists a constant $K>0$ such that for $\gamma \geqq K$

(i) $(L+\gamma I)^{-1}$ and $(M+\gamma I)^{-1}$ are compact operators defined on all of $\mathscr{B}$;

(ii) $(M+\gamma I)^{-1}-(L+\gamma I)^{-1}$ is a positive operator;

(iii) $(M+\gamma I)^{-1}$ is $v_{0}$-positive.

If $L u=\theta$ has a nontrivial solution $u_{0} \in \mathscr{P}$ and if $M u_{0} \neq \theta$, then no nontrivial solution of $M v=\theta$ belongs $\cdot$ to $\mathscr{P}$.

Proof. Defining $A=(L+\gamma I)^{-1}$ and $B=(M+\gamma I)^{-1}$, we have $A u_{0}=\gamma^{-1} u_{0}$ for some nonzero $u_{0} \in \mathscr{P}$. Writing

$$
\left(B u_{0}-u_{0} / \gamma\right)=(B-A) u_{0}+\left(A u_{0}-u_{0} / \gamma\right),
$$

we see by 2.5 that the maximal eigenvalue of $B$ satisfies $\beta \geqq 1 / \gamma$. Since $\beta=1 / \gamma$ only if $M u_{0}=\theta$, we have $\beta>1 / \gamma$. Therefore $v$ is a solution of $M v=\theta$ iff it satisfies $B v=\gamma^{-1} v$ with $\gamma^{-1}<\beta$. Since $B$ has only one eigenvector in $\mathscr{P}$ and this eigenvector corresponds to $\beta>1 / \gamma$, it follows that no nontrivial solution of $M v=\theta$ belongs to $\mathscr{P}$.

3. Application to elliptic equations. In this section we shall derive some properties of fundamental solutions of elliptic equations which appear to be of some interest in themselves but are primarily needed to apply the theory of $\$ 2$ to nonselfadjoint uniformly elliptic equations. The equations to be considered will be of the form

$$
L u \equiv-\sum_{i, j=1}^{n} \frac{\partial}{\partial x_{j}}\left(a_{i j} \frac{\partial u}{\partial x_{i}}\right)+\sum_{i=1}^{n} b_{i} \frac{\partial u}{\partial x_{i}}+c u=0
$$

whose coefficients are defined in a domain $\Omega \subset R^{n}$. As a specific application of $\$ 2$ to equations of the form $L u=0$, we shall generalize the following two propositions known to be valid for ordinary differential equations:

(i) If $u_{1}(x)$ and $u_{2}(x)$ are linearly independent solutions of

$$
-\left(a u^{\prime}\right)^{\prime}+b u^{\prime}+c u=0 ; \quad a(x)>0
$$

then the zeros of $u_{1}$ separate the zeros of $u_{2}$. 
(ii) If $u(x)$ and $v(x)$ are nontrivial solutions of

$$
\begin{aligned}
-\left(a u^{\prime}\right)^{\prime}+b u^{\prime}+c u & =0 ; \quad a(x)>0 \\
-\left(a v^{\prime}\right)^{\prime}+b v^{\prime}+(c-p) v & =0
\end{aligned}
$$

where $p(x)>0$, then the zeros of $v(x)$ separate the zeros of $u(x)$.

While comparison theorems for equations of the form (5) have been studied by Protter [5] and Swanson [6], these authors considered the more general case of two elliptic equations which are allowed to differ in all their coefficients. By restricting our attention to two equations of the form (5) which differ only in the last coefficient $c(x)$, we shall be able to derive a comparison theorem based on different hypotheses. (In the case of the ordinary differential equations (6) which differ only in the last coefficient, the corresponding simplification results from the fact that both equations can be put into selfadjoint form by multiplying through by the same factor: $\exp \left(-\int b / a d x\right)$.)

In addition to the formal operator $L$ defined in (5) we shall define a class of operators $L_{s}$ whose domains consist of sufficiently regular functions satisfying $\partial u / \partial v+s u=0$ on $\partial \Omega$. Here $\partial u / \partial v$ is the transverse derivative defined by

$$
\frac{\partial u}{\partial \nu}=\sum a_{i j} \frac{\partial u}{\partial x_{i}} \frac{\partial \nu}{\partial x_{j}}
$$

where $\partial v / \partial x_{j}$ denotes the cosine of the angle between the exterior normal to $\partial \Omega$ and the positive $x_{j}$-axis. The function $s(x)$ is to be piecewise smooth with values satisfying $-\infty<s(x) \leqq+\infty$, where $s(x)+\infty$ is used to denote the boundary condition $u(x)=0$. It is assumed throughout that the coefficients of $L$, the function $s(x)$, and the boundary of $\Omega$ are sufficiently regular to assure the existence of fundamental solutions for $\left(L_{s}+\gamma I\right) u=0$ for sufficiently large values of $\gamma$. That is, given $s(x)$ we assume the existence of a constant $K$ such that for $\gamma \geqq K$

$$
\left(L_{s}+\gamma I\right)^{-1} f=\int_{\Omega} G_{s}(x, \xi ; \gamma) f(\xi) d \xi
$$

defines a compact operator on $\mathscr{L}^{2}(\Omega)$. We shall make use of the fact that $G_{s}$ satisfies

$$
(L+\gamma I) G_{s}=0 \quad \partial G_{s} / \partial \nu+s G_{s}=0
$$

in the first variable for each fixed $\xi \in \Omega$ and $\left(L^{*}+\gamma I\right) G_{s}=0$ in the second variable for each fixed $x \in \Omega$.

If $s(x) \geqq 0$ on $\partial \Omega$ and $c(x)+\gamma \geqq 0$ in $\Omega$, then it follows readily from the maximum principle that $G_{s}(x, \xi ; \gamma)$ is positive in $\Omega \times \Omega$. We shall require a similar result even if $s(x)$ is allowed to assume negative values.

3.1. TheOREM. Given $s(x)$ defined on $\partial \Omega$, there exists a constant $K$ such that for $\gamma \geqq K$ the fundamental solution $G_{s}(x, \xi ; \gamma)$ satisfying (7) is positive in $\Omega \times \Omega$. 
Proof. Define

$$
\begin{aligned}
\mathscr{D}[\varphi] & =\int_{\Omega}\left[\sum a_{i j} \frac{\partial \varphi}{\partial x_{i}} \frac{\partial \varphi}{\partial x_{j}}+c \varphi^{2}\right] d x \\
\eta_{\gamma}[\varphi] & =\int_{\Omega}\left[\sum a_{i j} \frac{\partial \varphi}{\partial x_{i}} \frac{\partial \varphi}{\partial x_{j}}+\varphi \sum b_{i} \frac{\partial \varphi}{\partial x_{i}}+(c+\gamma) \varphi^{2}\right] d x
\end{aligned}
$$

for all $\varphi(x)$ which are continuous and have piecewise continuous first partial derivatives in $\Omega$ and satisfy $\int_{\Omega} \varphi^{2} d x=1$. Noting that

$$
\eta_{\gamma}[\varphi]=\frac{1}{2}(\mathscr{D}[\varphi]+\gamma)+\frac{1}{2} \int_{\Omega}\left[\sum a_{i j} \frac{\partial \varphi}{\partial x_{i}} \frac{\partial \varphi}{\partial x_{j}}+2 \varphi \sum b_{i} \frac{\partial \varphi}{\partial x_{i}}+(c+\gamma) \phi^{2}\right] d x
$$

we can choose $K_{1}$ sufficiently large such that for $\gamma \geqq K_{1}$ the matrix

$$
\left(\begin{array}{llll}
a_{11} & \cdots & a_{1 n} & b_{1} \\
\vdots & & \vdots & \vdots \\
a_{n 1} & \cdots & a_{n n} & b_{n} \\
b_{1} & \cdots & b_{n} & c+\gamma
\end{array}\right)
$$

is positive definite. For such a choice of $\gamma$ we clearly have $\eta_{\gamma} \geqq \frac{1}{2}(\mathscr{D}[\varphi]+\gamma)$. An inequality due to Courant [9] asserts the existence of positive constants $C$ and $C^{\prime}$, depending only on the coefficients of $L$ and on $s(x)$, such that

$$
\left|\int_{\partial \Omega} s \varphi^{2} d x\right|<C(\mathscr{D}[\varphi])^{1 / 2}+C^{\prime}
$$

for all admissible $\varphi$. Given $C$ and $C^{\prime}$ we can choose $K \geqq K_{1}$ such that for $\gamma \geqq K$

$$
\eta_{y}[\varphi] \geqq \frac{1}{2}(\mathscr{D}[\varphi]+\gamma) \geqq C(\mathscr{D}[\varphi])^{1 / 2}+C^{\prime} .
$$

Suppose now that $\gamma \geqq K$ and that $G_{s}(x, \xi ; \gamma)$ becomes negative in $\Omega \times \Omega$. This implies the existence of $\xi_{0} \in \Omega$ such that $G_{s}\left(x, \xi_{0} ; \gamma\right)<0$ in a subdomain $\Omega_{0}$ of $\Omega$ which is bounded by nodal surfaces of $G_{s}\left(x, \xi_{0} ; \gamma\right)$ and perhaps segments of $\partial \Omega$. Since $\lim _{x \rightarrow \xi_{0}} G_{s}\left(x, \xi_{0} ; \gamma\right)=+\infty, G_{s}$ is regular in $\Omega_{0}$ and the function

$$
\begin{aligned}
\varphi(x) & =k G_{s}\left(x, \xi_{0} ; \gamma\right) \text { in } \Omega_{0}, \\
& =0 \text { in } \Omega-\Omega_{0}
\end{aligned}
$$

is made admissible in Courant's inequality by choosing the constant $k$ such that

$$
\int_{\Omega} \varphi^{2} d x=k^{2} \int_{\Omega_{0}} G_{s}^{2}\left(x, \xi_{0} ; \gamma\right) d x=1 .
$$

By Green's theorem and the characteristic properties of $G_{s}$,

$$
\begin{aligned}
\eta_{\gamma}[\varphi] & =k^{2} \int_{\Omega_{0}}\left[\sum a_{i j} \frac{\partial G_{s}}{\partial x_{i}} \frac{\partial G_{s}}{\partial x_{j}}+\sum b_{i} \frac{\partial G_{s}}{\partial x_{i}} G_{s}+(c+\gamma) G_{s}^{2}\right] d x \\
& =k^{2} \int_{\Omega_{0}} G_{s}(L+\gamma I) G_{s} d x+k^{2} \int_{\partial \Omega_{0} \cap \partial \Omega} G_{s} \frac{\partial G_{s}}{\partial \nu} d \sigma .
\end{aligned}
$$


Since $(L+\gamma I) G_{s}\left(x, \xi_{0} ; \gamma\right)=0$ and $\partial G_{s} / \partial \nu=-s G_{s}=-s \varphi / k$ on $\partial \Omega_{0} \cap \partial \Omega$,

$$
\eta_{\gamma}[\varphi] \leqq\left|\int_{\partial \Omega} s \varphi^{2} d \sigma\right|
$$

and by $(8), \eta_{\gamma}[\varphi]<C(\mathscr{D}[\varphi])^{1 / 2}+C^{\prime}$. But this contradicts (9) and shows that $G_{s}(x, \xi ; \gamma)$ is nonnegative in $\Omega \times \Omega$. That $G_{s}\left(x, \xi_{0} ; \gamma\right)$ is actually positive for any $\xi_{0} \in \Omega$ follows from the fact that for sufficiently large values of $\gamma$ the Hopf maximum principle applies to solutions of $(L+\gamma I) u=0$. Therefore if $c\left(x_{0}\right)+\gamma>0$ and $G\left(x_{0}, \xi_{0} ; \gamma\right)=0$ for some $\left(x_{0}, \xi_{0}\right) \in \Omega \times \Omega$, then $G\left(x, \xi_{0} ; \gamma\right)$ must change sign at $x=x_{0}$.

From Theorem 3.1 it follows that if $\mathscr{B}$ is $\mathscr{L}^{2}(\Omega)$ and $\mathscr{P}$ is the cone of nonnegative functions in $\mathscr{B}$ then, for sufficiently large values of $\gamma,(L+\gamma I)^{-1}$ is a positive operator. The following theorem asserts the stronger result that for sufficiently large $\gamma$, $(L+\gamma I)^{-1}$ is $u_{0}$-positive.

3.2. ThEOREM. Let $\mathscr{B}$ be the Banach space $\mathscr{L}^{2}(\Omega)$ and let $\mathscr{P}$ denote the cone of elements in $\mathscr{B}$ which are nonnegative on $\Omega$. Given a fixed function $s(x),-\infty<s \leqq \infty$, there exists a $K>0$ such that for $\gamma \geqq K,\left(L_{s}+\gamma I\right)^{-1}$ is $u_{0}$-positive.

Proof. Our proof involving the fundamental solution $G_{s}(x, \xi ; \gamma)$ of (7) will be a generalization of a similar result of Krasnosel'skir and Sobolevskiir [11] for the Green's function $G_{\infty}(x, \xi ; \gamma)$ (see also [8, p. 232]). We choose $K$ sufficiently large so that $c(x)+K>1$ in $\Omega$ and such that $G_{s}$ exists and is positive in $\Omega \times \Omega$ for $\gamma \geqq K$. It is shown in [8, p. 258] that for $\gamma \geqq K$ the $u_{0}$-positivity of $(L+\gamma I)^{-1}$ follows from the following property of $G_{s}(x, \xi ; \gamma)$ : For every domain $\Omega_{0}$ of positive measure which satisfies $\bar{\Omega}_{0} \subset \Omega$ there exists a positive $\varepsilon$, depending only on $\Omega_{0}$, such that

$$
\varepsilon \int_{\Omega} G_{s}(x, \xi ; \gamma) d \xi \leqq \int_{\Omega_{0}} G_{s}(x, \xi ; \gamma) d \xi
$$

To verify the existence of such an $\varepsilon$ we define $\varphi_{0}(x) \equiv 1$ in $\bar{\Omega}$ and a continuous function

$$
\begin{aligned}
\varphi_{1}(x) & \equiv 0 \quad \text { in } \bar{\Omega}-\Omega_{0}, \\
& \equiv 1 \quad \text { in some } \bar{\Omega}_{1} \subset \Omega_{0}
\end{aligned}
$$

and $0 \leqq \varphi_{1}(x) \leqq 1$ elsewhere. The functions

$$
\Psi_{0}(x)=\int_{\Omega} G_{s}(x, \xi ; \gamma) \varphi_{0}(\xi) d \xi
$$

and

both satisfy

$$
\Psi_{1}(x)=\int_{\Omega} G_{s}(x, \xi ; \gamma) \varphi_{1}(\xi) d \xi
$$

$$
\begin{aligned}
(L+\gamma) \Psi_{i} & =\varphi_{i}, \quad i=0,1 \\
\partial \Psi_{i} / \partial \nu+s \Psi_{i} & =0 \quad \text { on } \partial \Omega ; \quad i=0,1 .
\end{aligned}
$$


Furthermore $\Psi_{1}>0$ in $\bar{\Omega}-\{x \in \partial \Omega \mid s(x)=+\infty\}$, and by the Hopf maximum principle, $\partial \Psi_{i} / \partial \nu<0$ on $\Gamma_{1} \equiv\{x \in \partial \Omega \mid s(x)=+\infty\}$. Since $\Gamma_{1}$ is compact we can find $\varepsilon_{0}>0$ such that $\Psi_{\varepsilon}(x) \equiv \Psi_{1}(x)-\varepsilon_{0} \Psi_{0}(x)$ has a negative exterior normal derivative for all $x \in \Gamma_{1}$. At points of $\bar{\Omega}$ at a small positive distance from $\Gamma_{1}, \Psi_{\varepsilon}(x)$ will clearly be positive. Furthermore at points of $\bar{\Omega}$ which are a positive distance from $\Gamma_{1}$ $G_{s}(x, \xi)$ is bounded below by a positive constant, so that $\Psi_{0}$ and $\Psi_{1}$ are strictly positive and bounded on $\bar{\Omega}-\Gamma_{1}$. Therefore we can choose $\varepsilon_{1} \leqq \varepsilon_{0}$ sufficiently small so that $\Psi_{\varepsilon}(x)$ is positive everywhere in $\bar{\Omega}$ whenever $\varepsilon \leqq \varepsilon_{1}$. For such values of $\varepsilon$

$$
\varepsilon \int_{\Omega} G_{s}(x, \xi ; \gamma) d \xi \leqq \int_{\Omega} G_{s}(x, \xi ; \gamma) \varphi_{1}(\xi) d \xi
$$

for all $x \in \bar{\Omega}$. But since $G_{s}$ is nonnegative,

$$
\int_{\Omega} G_{s}(x, \xi ; \gamma) \varphi_{1}(\xi) d \xi \leqq \int_{\Omega_{0}} G_{s}(x, \xi ; \gamma) d \xi
$$

which completes the proof.

3.3. SePARATION TheOREM. Let $u_{1}(x)$ and $u_{2}(x)$ be nontrivial solutions of $L u=0$ and let $\Omega$ be a nodal domain for $u_{1}$. Then $u_{2}$ changes sign in $\Omega$ or else $u_{2}$ is a constant multiple of $u_{1}$.

Proof. Suppose $u_{2}(x)$ satisfies $\partial u_{2} / \partial v+s u_{2}=0$ on $\partial \Omega$ and choose $\gamma$ sufficiently large so that $\left(L_{\infty}+\gamma I\right)^{-1}$ and $\left(L_{s}+\gamma I\right)^{-1}$ are $u_{0}$-positive and $c(x)+\gamma>0$. In order to apply Theorem 2.6 it suffices to show that $G_{s}(x, \xi ; \gamma) \geqq G_{\infty}(x, \xi ; \gamma)$ in $\Omega \times \Omega$. To that end, suppose $G_{s}\left(x_{0}, \xi_{0} ; \gamma\right)<G_{\infty}\left(x_{0}, \xi_{0} ; \gamma\right)$. Then there exists a subdomain $\Omega_{0} \subset \Omega$ in which $G_{s}\left(x, \xi_{0}\right)<G_{\infty}\left(x, \xi_{0}\right)$ and such that $G_{s}-G_{\infty}$ vanishes on $\partial \Omega_{0}$. Since $G_{s}$ and $G_{\infty}$ have the same fundamental singularity, $G_{\infty}\left(x, \xi_{0} ; \gamma\right)-G_{s}\left(x, \xi_{0} ; \gamma\right)$ is a regular solution of $(L+\gamma I) u=0$ in $\Omega_{0}$ which vanishes on $\partial \Omega_{0}$. By the maximum principle, this implies that $G_{\infty}\left(x, \xi_{0} ; \gamma\right)-G_{s}\left(x, \xi_{0} ; \gamma\right) \equiv 0$ in $\Omega_{0}$. Therefore $G_{s} \geqq G_{\infty}$ in $\Omega \times \Omega$.

Now according to Theorem 2.6 either $u_{2}$ changes sign in $\Omega$ or else $L_{\infty} u_{2}=0$. In the latter case $u_{2}=0$ on $\partial \Omega$ and $u_{1}$ and $u_{2}$ are both positive eigenfunctions of $\left(L_{\infty}+\gamma I\right)^{-1}$. Since the maximal eigenvalue of $\left(L_{\infty}+\gamma I\right)^{-1}$ is simple, $u_{1} / u_{2}=$ constant.

3.4. Comparison TheORem. Let $u(x)$ be a nontrivial solution of $L u=0$ and let $\Omega$ be a nodal domain for $u$. If $p(x) \geqq 0$ in $\Omega(p(x) \not \equiv)$ and $v(x)$ is a nontrivial solution of $L v-p v=0$, then $v(x)$ changes sign in $\Omega$.

Proof. Suppose $v(x)$ satisfies $\partial v / \partial \nu+s v=0$ on $\partial \Omega$ and let $H_{s}(x, \xi ; \gamma)$ denote the fundamental solution of $\left(L_{s}-p I+\gamma I\right) v=0$. Let $\gamma$ be chosen sufficiently large so that $H_{s}(x, \xi ; \gamma)$ and $G_{\infty}(x, \xi ; \gamma)$ are positive in $\Omega \times \Omega$ and such that $\left(L_{s}-p I+\gamma I\right)^{-1}$ and $\left(L_{\infty}+\gamma I\right)^{-1}$ are $u_{0}$-positive. 
In order to apply Theorem 2.6 we need only show that $H_{s} \geqq G_{\infty}$ in $\Omega \times \Omega$. By the same technique used in the preceding theorem, it follows that $H_{s} \geqq H_{\infty}$. It therefore remains to show that $H_{\infty} \geqq G_{\infty}$. To see this we apply the identity

$$
\int_{\Omega_{r}}\left(v L u-u L^{*} v\right) d y=\int_{\partial \Omega_{r}}\left[u \frac{\partial v}{\partial \nu}-v \frac{\partial u}{\partial \nu}+\sum b_{i} \frac{\partial v}{\partial x_{i}} u v\right] d \sigma
$$

to the functions $v(y)=G_{\infty}(x, y ; \gamma)$ and $u(y)=H_{\infty}(y, \xi ; \gamma)$ where $\Omega_{r}$ is the domain $\Omega$ with spheres of radius $r$ about $y=x$ and $y=\xi$ deleted. As $r \rightarrow 0$ we obtain (for $\gamma$ as above)

$$
\int_{\Omega}\left[G_{\infty}(x, y) L H_{\infty}(y, \xi)-H_{\infty}(y, \xi) L^{*} G_{\infty}(x, y)\right] d y=H_{\infty}(x, \xi)-G_{\infty}(x, \xi) .
$$

On the other hand, since $L H_{\infty}=(p-\gamma) H_{\infty}$ and $L^{*} G_{\infty}=-\gamma G_{\infty}$, the integral on the left side of (10) reduces to $\int_{\Omega} p G_{\infty} H_{\infty} d y$ and we obtain

$$
H_{\infty}=G_{\infty}+\int_{\Omega} p G_{\infty} H_{\infty} d y \geqq G_{\infty} .
$$

Therefore Theorem 2.6 applies. Since $p \not \equiv 0$ we can not have $L_{\infty} v=0$, and it follows that $v(x)$ changes sign in $\Omega$.

According to Theorem 3.1, for sufficiently large $\gamma, G_{\infty}(x, \xi ; \gamma)$ is positive in $\Omega \times \Omega$. As a final remark we shall show that Theorems 3.2 and 3.4 can be used to characterize the appropriate range of values for $\gamma$ to assure the positivity of the Green's function.

Choosing $\gamma_{0}$ sufficiently large so that $\left(L_{\infty}+\gamma_{0} I\right)^{-1}$ has property $\left(^{*}\right)$, we know that $\left(L_{\infty}+\gamma_{0} I\right)^{-1}$ has a maximal eigenvalue $\alpha_{0}$ and that the eigenvalues of $\left(L_{\infty}+\left(\gamma_{0}+\alpha_{0}\right) I\right)^{-1}$ all satisfy $\operatorname{Re}(\lambda)>0$. By the spectral mapping theorem it follows that $L_{\infty}$ has a minimal real eigenvalue $\lambda_{0}=1 / \alpha_{0}-\gamma_{0}$ whose eigenfunction may be taken positive in $\Omega$ and which satisfies $\lambda_{0}<\operatorname{Re}(\lambda)$ for all other eigenvalues of $L_{\infty}$.

3.5. THEOREM. If $\gamma>-\lambda_{0}$, then $G_{\infty}(x, \xi ; \gamma)$ is positive in $\Omega \times \Omega$.

Proof. Suppose that $G_{\infty}\left(x_{0}, \xi_{0} ; \gamma\right)<0$ for some fixed $\gamma>-\lambda_{0}$. Then there exists a subdomain $\Omega_{0} \subset \Omega$ such that $G_{\infty}\left(x, \xi_{0} ; \gamma\right)<0$ for $x$ in $\Omega_{0}$ and for which $G_{\infty}\left(x, \xi_{0} ; \gamma\right)$ is a regular solution of

$$
\left(L_{\infty}+\gamma I\right) G_{\infty}=0 \quad \text { in } \Omega_{0}, \quad G_{\infty}=0 \text { on } \partial \Omega_{0} .
$$

According to Theorem 3.4, every solution of $\left(L_{\infty}-\lambda_{0} I\right) v=0$ must change sign in $\Omega_{0}$. But this contradicts the existence of a positive eigenfunction satisfying $L_{\infty} v=\lambda v_{0}$ and shows that $G_{\infty}$ is nonnegative in $\Omega \times \Omega$. The fact that $G_{\infty}$ is actually positive follows from the local validity at interior points of $\Omega$ of the Hopf maximum principle (as described in [13]).

Added in proof. The author recently learned that the first proof of a comparison theorem for selfadjoint second order elliptic equations was given by Mauro Picone in Un teorema sulle soluzioni delle equazioni lineari ellitiche autoaggiunte alle 
derivate parziali del secondo-ordine, Atti Accad. Naz. Lincei 20 (1911), 213-219. This proof is based on a generalization of the Picone Identity for ordinary differential equations and is closely related to the transformation used by Clark and Swanson [3].

\section{BIBLIOGRAPHY}

1. P. Hartman and A. Wintner, On a comparison theorem for self-adjoint equations of elliptic type, Proc. Amer. Math. Soc. 6 (1955), 862-865.

2. K. Kreith, A new proof of a comparison theorem for elliptic equations, Proc. Amer. Math. Soc. 14 (1963), 33-35.

3. C. Clark and C. A. Swanson, Comparison theorems for elliptic differential equations, Proc. Amer. Math. Soc. 16 (1965), 886-890.

4. K. Kreith, A strong comparison theorem for selfadjoint elliptic equations, Proc. Amer. Math. Soc. 19 (1968), 989-990.

5. M. H. Protter, A comparison theorem for elliptic equations, Proc. Amer. Math. Soc. 10 (1959), 296-299.

6. C. A. Swanson, A comparison theorem for elliptic differential equations, Proc. Amer. Math. Soc. 17 (1966), 611-616.

7. C. A. Swanson, Comparison theorems for elliptic equations on unbounded domains, Trans. Amer. Math. Soc. 126 (1967), 278-285.

8. M. A. Krasnosel'skiī, Positive solutions of operator equations, Noordhoff, Groningen, 1964.

9. R. Courant, Über die Eigenwerte bei den Differentialgleichungen der mathematischen Physik, Math. Z. 7 (1920), 1-57.

10. S. Bergman and M. Schiffer, Kernel functions and elliptic differential equations in mathematical physics, Acàdemic Press, New York, 1953.

11. M. A. Krasnosel'skiĭ and P. E. Sobolevskiĭ, On the non-negative characteristic function of a first boundary value problem for an elliptic equation, Uspehi Mat. Nauk 16 (1961).

12. E. Hopf, A remark on linear elliptic differential equations of second order, Proc. Amer. Math. Soc. 3 (1952), 791-793.

13. K. Kreith, A remark on a comparison theorem of Swanson, Proc. Amer. Math. Soc. 20 (1969), 549-550.

\section{UNiversity of CALIFORNIA,}

Davis, CALIFornia 